TRANSACTIONS OF THE

AMERICAN MATHEMATICAL SOCIETY

Volume 350, Number 6, June 1998, Pages 2349-2365

S $0002-9947(98) 02159-X$

\title{
STRONGLY INDEFINITE SYSTEMS WITH CRITICAL SOBOLEV EXPONENTS
}

\author{
JOSEPHUS HULSHOF, ENZO MITIDIERI, AND ROBERTUS VANDERVORST
}

\begin{abstract}
We consider an elliptic system of Hamiltonian type on a bounded domain. In the superlinear case with critical growth rates we obtain existence and positivity results for solutions under suitable conditions on the linear terms. Our proof is based on an adaptation of the dual variational method as applied before to the scalar case.
\end{abstract}

\section{INTRODUCTION}

Existence and non-existence of solutions of semilinear elliptic systems has been a subject of active research recently; see for example [FF], [L3], [PS]. Such systems are called variational if solutions can be viewed as critical points of an associated functional defined on a suitable function space. Restricting our attention to systems with two unknowns, we distinguish two classes of variational elliptic systems:

(a) potential systems;

(b) Hamiltonian systems.

Potential systems are of the form

$$
-L_{1} u=\frac{\partial H}{\partial u}(u, v), \quad \pm L_{2} v=\frac{\partial H}{\partial v}(u, v),
$$

where $L_{1}$ and $L_{2}$ are second order selfadjoint elliptic operators. Formally these are the Euler-Lagrange equations of the functional

$$
f(u, v)=\left(L_{1} u, u\right) \pm\left(L_{2} v, v\right)-\mathcal{H}(u, v) .
$$

Here $(\cdot, \cdot)$ is the $L^{2}$ inner product for functions defined on the underlying domain, and $\mathcal{H}(u, v)=\int H(u(x), v(x)) d x$. The functional $f$ is often called the Lagrangian. Its critical points correspond to weak solutions of $(A)$. The appropriate choice of the function spaces for $u$ and $v$ follows by requiring that the quadratic part of $f$ be well defined. This naturally leads to Sobolev spaces with square integrable first derivatives. Since also $\mathcal{H}(\cdot, \cdot)$ must be well defined, by virtue of the embedding theorems, some growth restrictions on this latter function are required. An extra difficulty appears if we consider system $(A)$ with a plus sign in the second equation, for then the corresponding function $f$ has a strongly indefinite quadratic part.

For systems of superlinear type and with subcritical growth the Mountain Pass Theorem $[\mathrm{AR}]$ or, in the strongly indefinite case, the Benci-Rabinowitz Theorem

Received by the editors June 5, 1996.

1991 Mathematics Subject Classification. Primary 35J50, 35J55, 35J65.

Key words and phrases. Elliptic variational systems, strongly indefinite functionals, dual method, critical Palais-Smale level, critical points, ground states, decay rates, scaling. 
$[\mathrm{BR}],[\mathrm{H}]$ for strongly indefinite functionals may be applied under some extra technical assumptions on $H$. If we consider system $(A)$ with critical growth, in general the critical point theorems cannot be applied due to a lack of compactness. For some special systems, however, decoupling techniques can be used to reduce the problem to one equation with a nonlocal term, see for example [FM1], [MM], which can be treated using concentration compactness methods in the spirit of P.-L. Lions [L1], [L2]. We point out that even if the decoupling technique is not applicable, systems of type $(A)$ behave in a certain sense like scalar equations. This is essentially due to the weak coupling between the two equations, see e.g. [CFM2].

For Hamiltonian systems the situation is quite different because they are of the form

$$
-L v=\frac{\partial H}{\partial u}(u, v), \quad-L^{*} u=\frac{\partial H}{\partial v}(u, v)
$$

where $L^{*}$ is the adjoint operator of $L$. In this case the corresponding functional is given by

$$
f(u, v)=(u, L v)-\mathcal{H}(u, v) .
$$

The non-quadratic part is called the Hamiltonian. One sees that the coupling now also occurs in the quadratic part of $f$, and therefore it is much stronger than in the potential case. An immediate consequence is that there is no longer only one appropriate choice of function spaces $[\mathrm{HV} 1],[\mathrm{FF}]$, because there is a trade-off between $u$ and $v$. This accounts for a much richer structure and a rather different characterization of the concept of critical growth. Consider for example

$$
\begin{cases}-\Delta v=H_{u}(u, v) & \text { in } \Omega, \\ -\Delta u=H_{v}(u, v) & \text { in } \Omega, \\ u=v=0 & \text { on } \partial \Omega,\end{cases}
$$

where $\Delta$ is the Laplace operator and $\Omega$ a domain in $\mathbf{R}^{n}$. The following result has been proved in $[\mathrm{M}],[\mathrm{V} 1]$.

Theorem 1. Let $\Omega \subset \mathbf{R}^{n}$ with $n \geq 3$ be a bounded starshaped domain with smooth boundary. Let $H \in C^{1}\left(\mathbf{R}^{2} ; \mathbf{R}\right)$ be such that $H(0,0)=0$. If there exist $a \in \mathbf{R}$ such that

$$
\frac{n}{n-2} H(u, v) \leq a u H_{u}(u, v)+(1-a) v H_{v}(u, v), \quad \text { for all }(u, v) \in \mathbf{R}^{2},
$$

then problem $(C)$ has no positive solutions of class $C^{2}(\Omega) \cap C^{1}(\bar{\Omega})$.

In this paper we consider a special case of system $(C)$, namely

$$
\left(I_{p, q}\right) \begin{cases}-\Delta v=\lambda u+|u|^{p-1} u, & \text { in } \Omega, \\ -\Delta u=\mu v+|v|^{q-1} v, & \text { in } \Omega, \\ u=v=0, & \text { on } \partial \Omega,\end{cases}
$$

where $\lambda$ and $\mu$ are real numbers. When $\lambda=\mu=0$, the condition in Theorem 1 reduces to

$$
\frac{1}{p+1}+\frac{1}{q+1} \leq \frac{n-2}{n}
$$

However, for the critical case, more precisely, for

$$
\frac{1}{p+1}+\frac{1}{q+1}=\frac{n-2}{n}, \quad p, q>1
$$


(the critical hyperbola), there may exist positive solutions for certain positive values of $\lambda$ and $\mu$. We mention that the Rellich type identity proved in [M], [V1] (see also $[\mathrm{P}],[\mathrm{PS}])$, which is used in the proof of Theorem 1 for system $(C)$ with $p$ and $q$ on the critical hyperbola, reduces to

$$
\frac{n}{2} \frac{p-1}{p+1} \lambda \int_{\Omega} u^{2}+\frac{n}{2} \frac{q-1}{q+1} \mu \int_{\Omega} v^{2}=\int_{\partial \Omega} \frac{\partial u}{\partial \nu} \frac{\partial v}{\partial \nu}(x, \nu) .
$$

For positive solutions on a domain starshaped with respect to the origin, the right hand side of this identity is positive. Thus the existence of positive solutions is not obstructed by the identity if $\lambda$ and $\mu$ are positive.

The purpose of this paper is to obtain such existence results for solutions of system $\left(I_{p, q}\right)$ in the case that $n \geq 4$. Let us recall that if $\lambda=\mu, p=q, u=v$, system $\left(I_{p, q}\right)$ reduces to the scalar semilinear elliptic equation $-\Delta u=\lambda u+|u|^{p-1} u$ with critical exponent $p=\frac{n+2}{n-2}$. For $n \geq 4$ Brézis and Nirenberg [BrN] have shown that a positive solution exists if $0<\lambda<\lambda_{1}$, where $\lambda_{1}$ is the first eigenvalue of the Laplacian with zero Dirichlet boundary data. This solution is constructed as a mountain pass of the corresponding Lagrangian. Crucial in their arguments is the fact that the critical value in the mountain pass is strictly less than an explicit constant related to the Sobolev embedding $H_{0}^{1} \hookrightarrow L^{\frac{2 n}{n-2}}$. Below this constant the Lagrangian satisfies the Palais-Smale (PS) condition, which is always satisfied in the subcritical case $1<p<\frac{n+2}{n-2}$.

In the subcritical case with superquadratic Hamiltonian, i.e.

$$
\frac{1}{p+1}+\frac{1}{q+1}>\frac{n-2}{n}, \quad p, q>1, \quad n>2,
$$

positive solutions of $\left(I_{p, q}\right)$ exist as critical points of the strongly indefinite functional

(6) $f(u, v)=\int_{\Omega} \nabla u \nabla v-\frac{\lambda}{2} \int_{\Omega} u^{2}-\frac{\mu}{2} \int_{\Omega} v^{2}-\frac{1}{p+1} \int_{\Omega}|u|^{p+1}-\frac{1}{q+1} \int_{\Omega}|v|^{q+1}$,

provided $\lambda \mu<\lambda_{1}^{2}$. This is shown in [HV1] and [FF] by means of an indefinite functional theorem due to Benci and Rabinowitz [BR], and in [CFM1] and [PV] by topological methods. Besides a PS-condition, this theorem requires compactness of the gradient of the non-quadratic part of the above Lagrangian, which fails in the critical case (4). For the scalar case the Lagrangian is not strongly indefinite, and therefore this second condition is not needed in [BrN].

In dealing with the critical case we shall circumvent this complication by using a dual formulation originally due to Clarke and Ekeland [CE]. This approach was used by Ambrosetti and Struwe [AS] as an alternative for the methods in [BrN]. The dual method requires a strict convexity condition on the non-quadratic part of the Lagrangian. This allows for a relation between critical points of the functional and its dual. The main advantage is now that critical points of the dual functional of (6), despite its original indefinite character, can be obtained as mountain passes, provided that the corresponding critical value lies below a constant depending only on $p, q$ and $n$, which is related to the best Sobolev constant appearing in a naturally associated Sobolev inequality. To get below this constant the exact asymptotic behaviour of the regular radial ground states of system $\left(I_{p, q}\right)$ with $\lambda=\mu=0$ and $\Omega=\mathbf{R}^{n}$ as established in [HV2] is required.

Let us remark that we have chosen to restrict our attention to a very explicit system with only pure powers in the nonlinearities. The advantage is that, as in [AS], this allows a much smoother presentation of the results and proofs. Clearly 
generalization to a larger class of systems is possible, but this will not be a subject of the present paper.

Throughout this paper we shall assume without loss of generality that $p \geq q$. We parameterize the critical hyperbola by

$$
p=\frac{n+2+2 \alpha}{n-2-2 \alpha}, \quad q=\frac{n+2-2 \alpha}{n-2+2 \alpha}, \quad \alpha \in[0,1]
$$

Denoting the eigenvalues of the Laplacian with homogeneous Dirichlet boundary conditions by $\lambda_{k}(k=1,2, \ldots)$, our main result reads as follows:

Theorem 2. Let $\Omega \subset \mathbf{R}^{n}$ with $n \geq 4$ be a bounded domain with smooth boundary, and let $p \geq q>1$ (i.e. $\alpha \in[0,1)$ ). Assume $\lambda \mu \neq \lambda_{k}^{2}$ for all $k$ and let one of the following conditions hold.

$$
\begin{aligned}
& \alpha=0: \quad \lambda+\mu>0, \\
& 0<\alpha<\frac{n-2}{2(n-1)}: \quad\left\{\begin{array}{l}
\mu>0 \\
n>4+2 \alpha, \mu=0, \lambda>0 ;
\end{array}\right. \\
& \frac{n-2}{2(n-1)}<\alpha<1: \quad\left\{\begin{array}{l}
\mu>0 ; \\
n(n-4 \alpha-4)+4-4 \alpha^{2}>0, \mu=0, \lambda>0 ;
\end{array}\right. \\
& \alpha=\frac{n-2}{2(n-1)}:\left\{\begin{array}{l}
\mu>0 ; n^{2}-6 n+6>0 ; \\
\mu=0, \lambda>0 .
\end{array}\right.
\end{aligned}
$$

Then the system $\left(I_{p, q}\right)$ has at least one nontrivial solution $(u, v) \in\left(C^{2}(\Omega) \cap C^{1}(\bar{\Omega})\right)^{2}$.

Moreover if $0<\lambda, \mu$ with $0<\lambda \mu<\lambda_{1}^{2}$, then there exists a solution with positive components.

Throughout this paper we shall assume that $\alpha<1$, i.e. $q>1$. For $q=1$ and $\mu=0$ the system reduces to the semilinear biharmonic equation $\Delta^{2} u=\lambda u+|u|^{p-1} u$ in $\Omega$ with boundary conditions $u=\Delta u=0$ on $\partial \Omega$, which has been studied in [V2]. In what follows we shall assume that $n \geq 4$. The case $n=3$ is more delicate, as can be seen in [BrN], and will be studied elsewhere.

This paper is organized as follows. In section 1 we introduce the functional analytic framework for the dual variational formulation of $\left(I_{p, q}\right)$ in the spirit of Ambrosetti and Struwe. Section 2 contains the key lemma concerning the PalaisSmale condition. In section 3 we discuss the geometric properties of the dual functional. Finally, in section 4, we use the asymptotic estimates proved in [HV2] of the ground states for $\left(I_{p, q}\right)$ with $\lambda=\mu=0$ in $\mathbf{R}^{n}$, to push the energy level below the critical Palais-Smale level. This allows us to complete the proof of Theorem 2.

\section{VARiational FORMUlation AND THE DUAL FUnCTIONAL}

Let $n \geq 3$, and let $\Omega \subset \mathbf{R}^{n}$ be a bounded domain with smooth boundary. In this paper we denote the standard $L^{2} \times L^{2}$-duality pairing by $\langle\cdot, \cdot\rangle$.

As in [HV1] and [FF], weak solutions of $\left(I_{p, q}\right)$ correspond to critical points of the functional $f$ defined on a suitable member of a family of Sobolev interpolation spaces. For a precise description of these spaces in terms of Fourier series, see [HV1], [FF]. Let

$$
D(-\Delta)=H^{2}(\Omega) \cap H_{0}^{1}(\Omega)
$$


For $p$ and $q$ satisfying (4) and $\alpha \in[0,1]$ given by (7), define

$$
E_{\alpha}=D\left((-\Delta)^{\frac{1+\alpha}{2}}\right) \times D\left((-\Delta)^{\frac{1-\alpha}{2}}\right)
$$

Since

$$
i: E_{\alpha} \hookrightarrow X_{\alpha}=L^{p+1}(\Omega) \times L^{q+1}(\Omega)=L^{\frac{2 n}{n-2-2 \alpha}}(\Omega) \times L^{\frac{2 n}{n-2+2 \alpha}}(\Omega),
$$

it follows that $f \in C^{1}\left(E_{\alpha}\right)$. Denoting by $E_{\alpha}^{*}$ the dual of $E_{\alpha}$ with respect to the $L^{2} \times L^{2}$-duality $\langle\cdot, \cdot\rangle$, the operator

$$
\partial_{-\Delta}=\left(\begin{array}{cc}
0 & -\Delta \\
-\Delta & 0
\end{array}\right): E_{\alpha} \longrightarrow E_{\alpha}^{*}
$$

is in fact an isometry; see [HV1]. Alternatively, by (linear) regularity theory (Agmon-Douglis-Nirenberg estimates; see [HV1] again), we can also view it as an isomorphism

$$
\partial_{-\Delta}: Y_{\alpha} \stackrel{\text { def }}{=}\left(W^{2, \frac{q+1}{q}} \cap W_{0}^{1, \frac{q+1}{q}}\right) \times\left(W^{2, \frac{p+1}{p}} \cap W_{0}^{1, \frac{p+1}{p}}\right) \longrightarrow X_{\alpha}^{*}=L^{\frac{p+1}{p}} \times L^{\frac{q+1}{q}} .
$$

Consequently the same holds for

$$
A_{\lambda \mu}=\left(\begin{array}{cc}
-\lambda I & -\Delta \\
-\Delta & -\mu I
\end{array}\right)
$$

provided $\lambda \mu \neq \lambda_{k}^{2}$, where $\left\{\lambda_{k}\right\}$ are the eigenvalues of the Laplacian. The inverse of $A_{\lambda \mu}: E_{\alpha} \longrightarrow E_{\alpha}^{*}$ is denoted by $A_{\lambda \mu}^{-1}$ and the inverse of $A_{\lambda \mu}: Y_{\alpha} \longrightarrow X_{\alpha}^{*}$ by $B_{\lambda \mu}$.

Thus we have

$$
X_{\alpha}^{*} \stackrel{i^{*}}{\longrightarrow} E_{\alpha}^{*} \stackrel{A_{\lambda, \mu}^{-1}}{\longrightarrow} E_{\alpha} \stackrel{i}{\longrightarrow} X_{\alpha}, X_{\alpha}^{*} \stackrel{B_{\lambda \mu}}{\longrightarrow} Y_{\alpha} \stackrel{j}{\longrightarrow} E_{\alpha} \text { and } j \circ B_{\lambda \mu}=A_{\lambda, \mu}^{-1} \circ i^{*}
$$

where $j$ is a noncompact Sobolev embedding. Neither of the two compositions is compact. The first one is denoted by $K_{\lambda, \mu}=i \circ A_{\lambda, \mu}^{-1} \circ i^{*}$. It has the property that $K_{\lambda, \mu}^{*}=K_{\lambda, \mu}$. This is a direct consequence of the fact that $\left(A_{\lambda \mu}^{-1}\right)^{*}=A_{\lambda \mu}^{-1}$. One may think of this as a selfadjointness property of operators from a reflexive space $X$ embedded in $L^{2} \times L^{2}$ into the dual space $X^{*}$ (with respect to the $L^{2} \times L^{2}$-duality).

Writing (6) as

$$
f(z)=\frac{1}{2}\left\langle A_{\lambda, \mu} z, z\right\rangle-\mathcal{H}(i z), \quad f: E_{\alpha} \rightarrow \mathbf{R},
$$

where

$$
z=(u, v), \quad H(z)=\frac{1}{p+1}|u|^{p+1}+\frac{1}{q+1}|v|^{q+1}, \quad \mathcal{H}(i z)=\int_{\Omega} H(z),
$$

we have

$$
d f(z)=A_{\lambda, \mu} z-i^{*} d \mathcal{H}(i z) .
$$

We define the dual functional

$$
f^{*}: X_{\alpha}^{*} \rightarrow \mathbf{R}
$$

by

$$
f^{*}(w)=\mathcal{H}^{*}(w)-\frac{1}{2}\left\langle K_{\lambda, \mu} w, w\right\rangle,
$$

where $\mathcal{H}^{*}: X_{\alpha}^{*} \rightarrow \mathbf{R}$ is the Legendre transform of $\mathcal{H}: X_{\alpha} \rightarrow \mathbf{R}$, i.e.

$$
\mathcal{H}^{*}(w)=\max _{z \in X_{\alpha}}(\langle z, w\rangle-\mathcal{H}(z)) .
$$


In fact we have

$$
\mathcal{H}^{*}(w)=\int_{\Omega} H^{*}(w(x)) d x
$$

where $H^{*}: \mathbf{R}^{2} \rightarrow \mathbf{R}$ is the Legendre transform of $H: \mathbf{R}^{2} \rightarrow \mathbf{R}$. Thus

$$
\mathcal{H}^{*}(w)=\int_{\Omega}\left(\frac{p}{p+1}\left|w_{1}\right|^{\frac{p+1}{p}}+\frac{q}{q+1}\left|w_{2}\right|^{\frac{q+1}{q}}\right) .
$$

Since $H: \mathbf{R}^{2} \rightarrow \mathbf{R}$ is strictly convex, the same holds for $\mathcal{H}^{*}: X_{\alpha}^{*} \rightarrow \mathbf{R}$. It follows that both $d \mathcal{H}$ and $d \mathcal{H}^{*}$ are invertible, and

$$
d \mathcal{H} \circ d \mathcal{H}^{*}=I_{X_{\alpha}^{*}}, d \mathcal{H}^{*} \circ d \mathcal{H}=I_{X_{\alpha}} .
$$

If $w \in X_{\alpha}^{*}$ is a critical point of $f^{*}$, it follows that $z=A_{\lambda, \mu}^{-1} i^{*} w \in E_{\alpha}$ is a critical point of $f$. Indeed, we have

$$
\begin{aligned}
& d \mathcal{H}^{*}(w)=K_{\lambda, \mu} w \text { in } X_{\alpha} \\
& \quad \Rightarrow(d \mathcal{H})^{-1}(w)=K_{\lambda, \mu} w=i A_{\lambda, \mu}^{-1} i^{*} w=i z \text { in } X_{\alpha} \\
& \quad \Rightarrow w=d \mathcal{H}(i z) \text { in } X_{\alpha}^{*} \\
& \quad \Rightarrow i^{*} w=A_{\lambda, \mu} z=i^{*} d \mathcal{H}(i z) \text { in } E_{\alpha}^{*} .
\end{aligned}
$$

Conversely, if $z=(u, v)$ is a critical point of $f: E_{\alpha} \rightarrow \mathbf{R}$, regularity theory (see [HV1]) implies that $A_{\lambda, \mu} z \in X_{\alpha}^{*}$. Consequently we may obtain a critical point of $f^{*}: X_{\alpha}^{*} \rightarrow \mathbf{R}$ by setting $w=i^{*} w=A_{\lambda, \mu} z$. Thus the formulations are in fact equivalent.

\section{The Palais-Smale Condition}

In this section we verify that $f^{*}$ satisfies a Palais-Smale condition for sufficiently small energy levels. We recall that a sequence $x_{n}$ is called a Palais-Smale sequence for the function $f \in C^{1}(X)$ at the energy level $c$, if $d f\left(x_{n}\right) \rightarrow 0$ in $X^{*}$ and $f\left(x_{n}\right) \rightarrow c$ for some $c \in \mathbf{R}$. We use the following formulation of the Palais-Smale condition.

$(P S)_{c}\left\{\begin{array}{l}\text { Let }\left\{w_{n}\right\} \in X_{\alpha}^{*} \text { be a Palais-Smale sequence for } f^{*} \text { at the energy level c. } \\ \text { Then there exists a subsequence }\left\{w_{n_{k}}\right\} \text { converging to some } w \in X_{\alpha}^{*} .\end{array}\right.$

Note that the limit $w$ is a stationary point of $f^{*}$ and that its critical value equals $c$. In order to see for which values of $c$ (if any) the function $f^{*}$ satisfies $(P S)_{c}$, let us introduce (see also [BrN], [L1], [L2], [HV2]) the embedding constants

$$
K_{p, q}=\inf \left\{\|\Delta u\|_{\frac{q+1}{q}} ; u \in W^{2, \frac{q+1}{q}} \cap W_{0}^{1, \frac{q+1}{q}}(\Omega),\|u\|_{p+1}=1\right\} .
$$

Let $p \geq q \geq 1$ and $0 \leq \alpha<1$.

Lemma 2.1. Let $0 \leq \alpha<1$ and $n>2+2 \alpha$. Then $f^{*}$ satisfies $(P S)_{c}$ for all $c$ with

$$
c<\frac{2}{n} K_{p, q}^{\frac{n}{2}}
$$

Lemma 2.1 will be proved in four steps. In first step we prove that $(P S)_{c^{-}}$ sequences $w_{n}$ are bounded in $X_{\alpha}^{*}$.

In the second step we show that the sequence $z_{n}=B_{\lambda, \mu} w_{n}$ has the property that $d f\left(j z_{n}\right) \rightarrow 0$, not only in $E_{\alpha}^{*}$ but also in $X_{\alpha}^{*}$. In addition, the energy level is 
not affected: $f\left(j z_{n}\right) \rightarrow c$. One might view the sequence $z_{n}$ as a $(P S)_{c}$-sequence of $f: E_{\alpha} \rightarrow \mathbf{R}$ with respect to the $X_{\alpha}^{*}$-topology.

In the third step we prove that this sequence $z_{n}$ contains a subsequence strongly convergent in $X_{\alpha}$ (and by regularity also in $Y_{\alpha}$ ).

Finally in the fourth step we tie everything up in terms of the original sequence $w_{n}$.

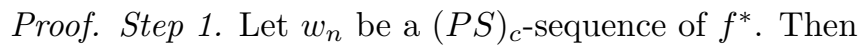

$$
f^{*}\left(w_{n}\right)-\frac{1}{2} d f^{*}\left(w_{n}\right) w_{n}=c+o(1)\left(\left\|w_{n}\right\|_{X_{\alpha}^{*}}+1\right) \text { as } n \rightarrow \infty,
$$

whence

$$
\int_{\Omega}\left|w_{1, n}\right|^{\frac{p+1}{p}}+\int_{\Omega}\left|w_{2, n}\right|^{\frac{q+1}{q}}=O(1)\left(\left\|w_{n}\right\|_{X_{\alpha}^{*}}+1\right) .
$$

Therefore $\left\|w_{n}\right\|_{X_{\alpha}^{*}} \leq C$.

Step 2. Let $z_{n}=B_{\lambda, \mu} w_{n} \in Y_{\alpha} \subset E_{\alpha}$. We note that we consider $z_{n}$ here as an element of $Y_{\alpha}$. As functions from $\Omega$ to $\mathbf{R}$ the elements $z_{n} \in Y_{\alpha}$ and $j z_{n} \in E_{\alpha}$ coincide. Since $d f^{*}\left(w_{n}\right)=d \mathcal{H}^{*}\left(w_{n}\right)-K_{\lambda, \mu} w_{n} \rightarrow 0$, i.e.

$$
K_{\lambda, \mu} w_{n}=d \mathcal{H}^{*}\left(w_{n}\right)+o(1) \text { in } X_{\alpha},
$$

by applying $d \mathcal{H}=\left(d \mathcal{H}^{*}\right)^{-1}$ and using

$$
K_{\lambda, \mu} w_{n}=i A_{\lambda, \mu}^{-1} i^{*} w_{n}=i j B_{\lambda, \mu} w_{n}=i j z_{n},
$$

we see that

$$
d \mathcal{H}\left(i j z_{n}\right)=B_{\lambda, \mu}^{-1} z_{n}+o(1) \text { in } X_{\alpha}^{*}
$$

This implies that

$$
i^{*} d \mathcal{H}\left(i j z_{n}\right)=i^{*} B_{\lambda, \mu}^{-1} z_{n}+o(1)=A_{\lambda, \mu} j z_{n}+o(1) \text { in } E_{\alpha}^{*},
$$

i.e. $d f\left(j z_{n}\right) \rightarrow 0$ in $E_{\alpha}^{*}$.

Next we show that $f\left(z_{n}\right) \rightarrow c$. At this point we no longer need to distinguish between $z_{n}$ and $j z_{n}$. Thus we may write

$$
\begin{aligned}
f\left(z_{n}\right) & =\frac{1}{2}\left\langle A_{\lambda, \mu} z_{n}, z_{n}\right\rangle-\mathcal{H}\left(i z_{n}\right) \\
& =\frac{1}{2}\left\langle i^{*} w_{n}, z_{n}\right\rangle-\mathcal{H}\left(i z_{n}\right) \\
& =\left\langle w_{n}, i z_{n}\right\rangle-\mathcal{H}\left(i z_{n}\right)-\frac{1}{2}\left\langle w_{n}, i z_{n}\right\rangle \\
& =\left\langle w_{n}, i z_{n}\right\rangle-\mathcal{H}\left(i z_{n}\right)-\frac{1}{2}\left\langle K_{\lambda, \mu} w_{n}, w_{n}\right\rangle \\
& =\left\langle w_{n}, d \mathcal{H}^{*}\left(w_{n}\right)\right\rangle-\mathcal{H}\left(d \mathcal{H}^{*}\left(w_{n}\right)\right)-\frac{1}{2}\left\langle K_{\lambda, \mu} w_{n}, w_{n}\right\rangle+o(1) \\
& =\mathcal{H}^{*}\left(w_{n}\right)-\frac{1}{2}\left\langle K_{\lambda, \mu} w_{n}, w_{n}\right\rangle+o(1) \\
& =c+o(1),
\end{aligned}
$$

which completes the proof of step 2 . 
Step 3. Since by step 1 the sequence $w_{n}$ is bounded in $X_{\alpha}^{*}$, it follows that the sequence $z_{n}$ is bounded both in $Y_{\alpha}$ and $E_{\alpha}$. Rewriting (2.3), we obtain

$$
\begin{cases}-\Delta v_{n}-\lambda u_{n}-u_{n}\left|u_{n}\right|^{p-1}=o(1) & \text { in } L^{\frac{p+1}{p}}, \\ -\Delta u_{n}-\mu v_{n}-v_{n}\left|v_{n}\right|^{q-1}=o(1) & \text { in } L^{\frac{q+1}{q}} .\end{cases}
$$

Thus along a subsequence we have that $z_{n} \rightarrow z$ in $Y_{\alpha}$. The weak limit is easily seen to be a weak solution of $\left(I_{p, q}\right)$, i.e. $d f(z)=0$. It remains to show that $f(z)=c$.

We first exclude the possibility that $z=0$. Since $\alpha<1$ we have $z_{n} \rightarrow z$ strongly in $L^{2} \times L^{2}$. Therefore for some nonnegative number $k$ we find that

$$
\int_{\Omega} \nabla u_{n} \nabla v_{n} \rightarrow k, \quad \int_{\Omega}\left|u_{n}\right|^{p+1} \rightarrow k, \quad \int_{\Omega}\left|v_{n}\right|^{q+1} \rightarrow k .
$$

Furthermore, from (2.5),

$$
\begin{aligned}
\int_{\Omega}\left|\Delta u_{n}\right|^{\frac{q+1}{q}} & =\int_{\Omega}\left|v_{n}^{q}\right| \operatorname{sign}\left(v_{n}^{q}\right)-\left|\Delta u_{n}\right|^{\frac{1}{q}} \operatorname{sign}\left(-\Delta u_{n}\right)+o(1) \\
& \leq\left(\int_{\Omega}\left|v_{n}\right|^{q+1}\right)^{\frac{q}{q+1}}\left(\int_{\Omega}\left|\Delta u_{n}\right|^{\frac{q+1}{q}}\right)^{\frac{1}{q+1}}+o(1)
\end{aligned}
$$

which yields

$$
\int_{\Omega}\left|\Delta u_{n}\right|^{\frac{q+1}{q}} \leq \int_{\Omega}\left|v_{n}\right|^{q+1}+o(1)=\int_{\Omega}\left|u_{n}\right|^{p+1}+o(1)=k+o(1) .
$$

On the other hand, by the Sobolev embedding theorem we have

$$
\|u\|_{p+1} \leq K_{p, q}^{-1}\|\Delta u\|_{\frac{q+1}{q}} .
$$

Combining the above inequality with (2.7) and taking the limit, we get

$$
k \geq K_{p, q}^{\frac{n}{2}}
$$

On the other hand, in view of (2.6) and step 2 we must have

$$
c \geq \frac{2}{n} k \geq \frac{2}{n} K_{p, q}^{\frac{n}{2}}
$$

This contradicts the assumption on $c$. Therefore the limit $z$ is nonzero.

Next we set (following [BrN]) $\alpha_{n}=u_{n}-u$ and $\beta_{n}=v_{n}-v$. Clearly $\left(\alpha_{n}, \beta_{n}\right) \rightarrow$ $(0,0)$ in $Y_{\alpha}$ and $\left(\alpha_{n}, \beta_{n}\right) \rightarrow(0,0)$ in $L^{2}$. In view of the Brézis-Lieb lemma (see $[\mathrm{BrL}])$ we have

$$
\int_{\Omega}\left|u_{n}\right|^{p+1}=\int_{\Omega}|u|^{p+1}+\int_{\Omega}\left|\alpha_{n}\right|^{p+1}+o(1),
$$

and likewise for $v$. Also

Hence

$$
\int_{\Omega} \nabla v_{n} \nabla u_{n}=\int_{\Omega} \nabla u \nabla v+\int_{\Omega} \nabla \alpha_{n} \nabla \beta_{n}+o(1) .
$$

As before, we may assume now that

$$
\int_{\Omega}\left|\alpha_{n}\right|^{p+1} \rightarrow k, \quad \int_{\Omega}\left|\beta_{n}\right|^{q+1} \rightarrow k, \quad \int_{\Omega} \nabla \alpha_{n} \nabla \beta_{n} \rightarrow k,
$$


so that

$$
f(z)+\frac{2}{n} \int_{\Omega} \nabla \alpha_{n} \nabla \beta_{n}=c+o(1) .
$$

This implies that either $k=0$ or $k \geq K_{p, q}^{\frac{n}{2}}$. Since by assumption $c<K_{p, q}^{\frac{n}{2}}$, it follows that $k=0$. This proves the strong convergence of $z_{n}$ in $X_{\alpha}$.

Step 4. Since $w_{n}=d \mathcal{H}\left(z_{n}\right)+o(1)$ in $X_{\alpha}^{*}$ and $z_{n}$ contains a strongly convergent subsequence, the existence of a convergent subsequence of $w_{n}$ in $X_{\alpha}^{*}$ is now immediate. This concludes the proof of Lemma 2.1.

\section{Geometry of the duAl FUnCtional}

In this section we begin with the construction of a critical point of mountain pass type of the dual functional $f^{*}$ defined by (1.8). Note that this functional has a local minimum at zero. There exist (see e.g. [BF], [CV1]) numbers $a, \rho$ such that

$$
f^{*}(w) \geq a>0, \quad \forall w \in X_{\alpha}=L^{\frac{p+1}{p}}(\Omega) \times L^{\frac{q+1}{q}}(\Omega) \text { with }\|w\|=\rho .
$$

Depending on the sign of the quadratic part, we see that far away from the origin we may find arbitrarily large positive and negative values, so that we are in a situation where we may apply the Mountain Pass Lemma. We now look for a curve connecting the origin to a point where the functional is negative and such that along this curve the functional has a maximum strictly less then the value in Lemma 2.1. Let $\left(w_{1}, w_{2}\right) \in X_{\alpha}^{*}$ be a function pair to be determined later.

Substituting $w=\left(t w_{1}, s w_{2}\right)$ in (1.8), we find that $f^{*}$ becomes

$$
f^{*}\left(t w_{1}, s w_{2}\right)=\frac{p t^{\frac{p+1}{p}}}{p+1} \int_{\Omega}\left|w_{1}\right|^{\frac{p+1}{p}}+\frac{q s^{\frac{q+1}{q}}}{q+1} \int_{\Omega}\left|w_{2}\right|^{\frac{q+1}{q}}-\frac{1}{2}\left\langle w, K_{\lambda \mu} w\right\rangle .
$$

Note that the last term in (3.1) contains $t$ and $s$. For fixed $w_{1}$ and $w_{2}$ the numbers $t$ and $s$ parameterize a linear subspace spanned by $\left(w_{1}, 0\right)$ and $\left(0, w_{2}\right)$. In this subspace the restricted functional has a saddle point. Roughly speaking, we are going to choose our curve through this point along the unstable direction.

The choice of $w_{1}$ and $w_{2}$ will have to ensure that along this curve the functional remains below the critical Palais-Smale level. The existence of a critical point of mountain pass type will follow from a standard min-max argument. For this purpose we need the precise decay rates [HV2] of the ground states of $\left(I_{p, q}\right)$ with $\lambda=\mu=0$ and $(p, q)$ on the critical hyperbola. Let us denote this one-parameter family of ground states by $z_{\epsilon}=\left(u_{\epsilon}, v_{\epsilon}\right)$, where $\epsilon \in(0, \infty)$. Replacing $\Omega$ by $\mathbf{R}^{n}$, putting $w_{1}=u_{\epsilon}^{p}$ and $w_{2}=v_{\epsilon}^{q}$ and taking $\lambda=\mu=0$, we find that (3.1) becomes

$$
g(t, s)=K_{p, q}^{\frac{n}{2}}\left(\frac{p}{p+1} t^{\frac{p+1}{p}}+\frac{q}{q+1} s^{\frac{q+1}{q}}-t s\right) .
$$

The function $g(t, s)$ has a saddle point in $(t, s)=(1,1)$, and

$$
g(1,1)=\frac{2}{n} K_{p, q}^{\frac{n}{2}},
$$

which is exactly the critical Palais-Smale level. There exists a curve through the origin along which $g(s, t)$ attains a maximum in $(1,1)$. Indeed this curve is given by

$$
t^{\frac{p+1}{p}}=s^{\frac{q+1}{q}} .
$$


Now we replace $\mathbf{R}^{n}$ by $\Omega$, and take arbitrary $\lambda$ and $\mu$. We will show that along (3.3) the function $f^{*}\left(t u_{\epsilon}^{p}, s v_{\epsilon}^{q}\right)$ has a smaller maximum. The parameter $\epsilon$ will be used to control the change from $\mathbf{R}^{n}$ to $\Omega$, whereas $\lambda$ and $\mu$, in combination with $\epsilon$ will push the maximum down below the critical Palais-Smale level.

We split (3.1) up into a part which resembles (3.2) and a remainder part. Let $\varphi_{\epsilon}$ and $\psi_{\epsilon}$ be the harmonic functions which coincide on the boundary of $\Omega$ with $u_{\epsilon}$ and $v_{\epsilon}$ respectively. Let $\Phi_{\epsilon}=\left(\varphi_{\epsilon}, \psi_{\epsilon}\right)$,

$$
D=\left(\begin{array}{cc}
s & 0 \\
0 & t
\end{array}\right) \text { and } \Lambda=\left(\begin{array}{cc}
\lambda & 0 \\
0 & \mu
\end{array}\right)
$$

First we deal with the quadratic part of the functional.

For $w_{\epsilon}=\left(t u_{\epsilon}^{p}, s v_{\epsilon}^{q}\right)$ we have

$$
K_{0,0} w_{\epsilon}=\left(\partial_{-\Delta}\right)^{-1} w_{\epsilon}=\left(\begin{array}{c}
s\left(u_{\epsilon}-\varphi_{\epsilon}\right) \\
t\left(v_{\epsilon}-\psi_{\epsilon}\right)
\end{array}\right)=D\left(z_{\epsilon}-\Phi_{\epsilon}\right)
$$

Therefore

$$
K_{\lambda, \mu} w_{\epsilon}=D\left(z_{\epsilon}-\Phi_{\epsilon}\right)+r_{\epsilon}
$$

By regularity the function pairs $K_{\lambda, \mu} w_{\epsilon}$ and $B_{\lambda, \mu} w_{\epsilon}$ coincide; thus

$$
\begin{aligned}
w_{\epsilon} & =B_{\lambda, \mu}^{-1} D\left(z_{\epsilon}-\Phi_{\epsilon}+r_{\epsilon}\right) \\
& =B_{\lambda, \mu}^{-1}\left(D\left(z_{\epsilon}-\Phi_{\epsilon}\right)\right)+B_{\lambda, \mu}^{-1}\left(r_{\epsilon}\right) \\
& =\partial_{-\Delta}\left(D\left(z_{\epsilon}-\Phi_{\epsilon}\right)\right)-\Lambda\left(D\left(z_{\epsilon}-\Phi_{\epsilon}\right)\right)+B_{\lambda, \mu}^{-1}\left(r_{\epsilon}\right) \\
& =w_{\epsilon}-\Lambda\left(D\left(z_{\epsilon}-\Phi_{\epsilon}\right)\right)+B_{\lambda, \mu}^{-1}\left(r_{\epsilon}\right)
\end{aligned}
$$

and

$$
r_{\epsilon}=B_{\lambda, \mu} \Lambda D\left(z_{\epsilon}-\Phi_{\epsilon}\right)=K_{\lambda, \mu} \Lambda D\left(z_{\epsilon}-\Phi_{\epsilon}\right) .
$$

For the quadratic part we now find that

$$
\begin{aligned}
\left\langle w_{\epsilon}, K_{\lambda \mu} w_{\epsilon}\right\rangle & =\left\langle w_{\epsilon}, D z_{\epsilon}-D \phi_{\epsilon}\right\rangle+\left\langle w_{\epsilon}, r_{\epsilon}\right\rangle \\
& =t s\left\{\int_{\Omega}\left(u_{\epsilon}-\varphi_{\epsilon}\right) u_{\epsilon}^{p}+\int_{\Omega}\left(v_{\epsilon}-\psi_{\epsilon}\right) v_{\epsilon}^{q}\right\}+\left\langle w_{\epsilon}, r_{\epsilon}\right\rangle .
\end{aligned}
$$

Combining (3.6) and (3.1), we get

$$
f^{*}\left(t w_{1}, s w_{2}\right)=g_{1}(t, s)+g_{2}(t, s),
$$

where

$$
\begin{aligned}
g_{1}(t, s)= & \frac{p t^{\frac{p+1}{p}}}{p+1} \int_{\Omega} u_{\epsilon}^{p+1}+\frac{q s^{\frac{q+1}{q}}}{q+1} \int_{\Omega} v_{\epsilon}^{q+1} \\
& -\frac{1}{2} t s \int_{\Omega} u_{\epsilon}^{p}\left(u_{\epsilon}-\varphi_{\epsilon}\right)-\frac{1}{2} t s \int_{\Omega} v_{\epsilon}^{q}\left(v_{\epsilon}-\psi_{\epsilon}\right) \\
= & \frac{p t^{\frac{p+1}{p}}}{p+1} A_{\epsilon}+\frac{q s^{\frac{q+1}{q}}}{q+1} B_{\epsilon}-t s C_{\epsilon},
\end{aligned}
$$

and

$$
g_{2}(t, s)=-\frac{1}{2}\left\langle w_{\epsilon}, r_{\epsilon}\right\rangle .
$$

We note that $C_{\epsilon}$ is the sum of two equal terms. Indeed, we have 


$$
\int_{\Omega} u_{\epsilon}^{p}\left(u_{\epsilon}-\varphi_{\epsilon}\right)=-\int_{\Omega}\left(\Delta\left(v_{\epsilon}-\psi_{\epsilon}\right)\right)\left(u_{\epsilon}-\varphi_{\epsilon}\right)=\int_{\Omega}\left(\nabla\left(u_{\epsilon}-\varphi_{\epsilon}\right), \nabla\left(v_{\epsilon}-\psi_{\epsilon}\right)\right),
$$

so that

$$
A_{\epsilon}=\int_{\Omega} u_{\epsilon}^{p+1}, \quad B_{\epsilon}=\int_{\Omega} v_{\epsilon}^{q+1}, \quad C_{\epsilon}=\int_{\Omega} u_{\epsilon}^{p}\left(u_{\epsilon}-\varphi_{\epsilon}\right)=\int_{\Omega} v_{\epsilon}^{q}\left(v_{\epsilon}-\psi_{\epsilon}\right) .
$$

A straightforward computation yields that along (3.3) the function $g_{1}$ has a maximum for

$$
t=\left(\frac{n}{n+2} \frac{\frac{p}{p+1} A_{\epsilon}+\frac{q}{q+1} B_{\epsilon}}{C_{\epsilon}}\right)^{\frac{p(q+1)}{p q-1}},
$$

and that this maximum is given by

$$
\begin{gathered}
M_{p, q}=\frac{2}{n}\left(\frac{n}{n+2}\right)^{\frac{n+2}{2}}\left(\frac{p}{p+1} A_{\epsilon}+\frac{q}{q+1} B_{\epsilon}\right)^{\frac{n+2}{2}} C_{\epsilon}^{-\frac{n}{2}} \\
=\frac{2}{n+2}\left(\frac{p}{p+1} A_{\epsilon}+\frac{q}{q+1} B_{\epsilon}\right)\left(\frac{\frac{p}{p+1} \frac{n}{n+2} A_{\epsilon}+\frac{q}{q+1} \frac{n}{n+2} B_{\epsilon}}{C_{\epsilon}}\right)^{\frac{n}{2}} .
\end{gathered}
$$

The second factor in (3.11) converges from below to $\frac{n+2}{n} K_{p, q}^{\frac{n}{2}}$ as $\epsilon \rightarrow 0$, whereas the third factor in (3.11) may be rewritten as

$$
\left(1+\frac{p}{p+1} \frac{n}{n+2} \frac{\int_{\Omega} u_{\epsilon}^{p} \varphi_{\epsilon}}{\int_{\Omega} u_{\epsilon}^{p}\left(u_{\epsilon}-\varphi_{\epsilon}\right)}+\frac{q}{q+1} \frac{n}{n+2} \frac{\int_{\Omega} v_{\epsilon}^{q} \psi_{\epsilon}}{\int_{\Omega} v_{\epsilon}^{q}\left(v_{\epsilon}-\psi_{\epsilon}\right)}\right)^{\frac{n}{2}} .
$$

Thus the maximum of $g_{1}$ along (3.3) satisfies

$$
\begin{gathered}
M_{p, q} \leq \frac{2}{n} K_{p, q}^{\frac{n}{2}}+O\left(\int_{\Omega} u_{\epsilon}^{p} \varphi_{\epsilon}\right)+O\left(\int_{\Omega} v_{\epsilon}^{q} \psi_{\epsilon}\right) \\
=\frac{2}{n} K_{p, q}^{\frac{n}{2}}+O\left(\left\|u_{\epsilon}\right\|_{p}^{p}\right) O\left(\left\|u_{\epsilon}\right\|_{L^{\infty}(\partial \Omega)}\right)+O\left(\left\|v_{\epsilon}\right\|_{q}^{q}\right) O\left(\left\|v_{\epsilon}\right\|_{L^{\infty}(\partial \Omega)}\right) .
\end{gathered}
$$

Next we consider $g_{2}$. Our goal is to show that as $\epsilon \rightarrow 0$, the contribution of $g_{2}$ will pull the maximum of $f^{*}\left(t w_{1}, s w_{2}\right)$ down along (3.3).

By (3.9), (3.4) and (3.5) we have

$$
\begin{aligned}
-2 g_{2}(t, s) & =\left\langle w_{\epsilon}, r_{\epsilon}\right\rangle=\left\langle w_{\epsilon}, K_{\lambda, \mu} \Lambda D\left(z_{\epsilon}-\Phi_{\epsilon}\right)\right\rangle=\left\langle K_{\lambda, \mu} w_{\epsilon}, \Lambda D\left(z_{\epsilon}-\Phi_{\epsilon}\right)\right\rangle \\
& =\left\langle D\left(z_{\epsilon}-\Phi_{\epsilon}\right)+r_{\epsilon}, \Lambda D\left(z_{\epsilon}-\Phi_{\epsilon}\right)\right\rangle \\
& =\left\langle D\left(z_{\epsilon}-\Phi_{\epsilon}\right), \Lambda D\left(z_{\epsilon}-\Phi_{\epsilon}\right)\right\rangle+\left\langle K_{\lambda, \mu} \Lambda D\left(z_{\epsilon}-\Phi_{\epsilon}\right), \Lambda D\left(z_{\epsilon}-\Phi_{\epsilon}\right)\right\rangle .
\end{aligned}
$$

Therefore

$$
\begin{gathered}
g_{2}(t, s)=-\frac{\lambda t^{2}}{2} \int_{\Omega} u_{\epsilon}^{2}-\frac{\mu s^{2}}{2} \int_{\Omega} v_{\epsilon}^{2}+\lambda t^{2} \int_{\Omega} u_{\epsilon} \varphi_{\epsilon} \\
+\mu s^{2} \int_{\Omega} v_{\epsilon} \psi_{\epsilon}-\frac{\lambda t^{2}}{2} \int_{\Omega} \varphi_{\epsilon}^{2}-\frac{\mu s^{2}}{2} \int_{\Omega} \psi_{\epsilon}^{2} \\
-\frac{1}{2}\left\langle K_{\lambda, \mu} \Lambda D\left(z_{\epsilon}-\Phi_{\epsilon}\right), \Lambda D\left(z_{\epsilon}-\Phi_{\epsilon}\right)\right\rangle .
\end{gathered}
$$


Using the boundedness of $K_{\lambda, \mu}: X_{\alpha}^{*} \longrightarrow X_{\alpha}$ and Hölder's inequality, (3.15) is estimated by

$$
\begin{aligned}
& \frac{1}{2}\left\|K_{\lambda, \mu}\right\|\left\|\Lambda D\left(z_{\epsilon}+\Phi_{\epsilon}\right)\right\|_{X_{\alpha}^{*}}^{2} \\
& \quad \leq\left\|K_{\lambda, \mu}\right\|\left(\lambda^{2} t^{2}\left(\left\|u_{\epsilon}\right\|_{\frac{p+1}{p}}^{2}+\left\|\varphi_{\epsilon}\right\|_{\frac{p+1}{p}}^{2}\right)+\mu^{2} s^{2}\left(\left\|v_{\epsilon}\right\|_{\frac{q+1}{q}}^{2}+\left\|\psi_{\epsilon}\right\|_{\frac{q+1}{q}}^{2}\right)\right) \\
& \quad \leq\left\|K_{\lambda, \mu}\right\|\left(\lambda^{2} t^{2}\left(\left\|u_{\epsilon}\right\|_{\frac{p+1}{p}}^{2}+\left\|u_{\epsilon}\right\|_{L^{\infty}(\partial \Omega)}^{2}\right)+\mu^{2} s^{2}\left(\left\|v_{\epsilon}\right\|_{\frac{q+1}{q}}^{2}+\left\|v_{\epsilon}\right\|_{L^{\infty}(\partial \Omega)}^{2}\right)\right) .
\end{aligned}
$$

This concludes the preliminary study of the estimates related to the geometric properties of the function $f^{*}$.

\section{SCALing the GRound States}

In this section we examine the limit behaviour of (3.12), (3.14) and (3.16) as $\epsilon \rightarrow 0$ in order to push the maximum of $f^{*}\left(t w_{1}, s w_{2}\right)$ along (3.3) below the critical Palais-Smale level. We recall that the ground states $\left(u_{\epsilon}, v_{\epsilon}\right)$ satisfy the following scaling property:

$$
u_{\epsilon}(x)=\epsilon^{-\frac{n}{p+1}} u_{1}\left(\frac{x}{\epsilon}\right), \quad v_{\epsilon}(x)=\epsilon^{-\frac{n}{q+1}} v_{1}\left(\frac{x}{\epsilon}\right)
$$

The asymptotic behaviour of $\left(u_{1}, v_{1}\right)$ is given by

Theorem 4.1 [HV2]. Let $p \geq \frac{n+2}{n-2}$. Let $\left(u_{1}, v_{1}\right)$ be a regular positive radial solution of $\left(I_{p, q}\right)$ on $R^{n}$ with $\lambda=\mu=0$. Then there exist constants $a>0$ and $b>0$, depending on $p$ and $n$, such that

$$
\lim _{r \rightarrow \infty} r^{n-2} v_{1}(r)=b
$$

and

$$
\begin{gathered}
\lim _{r \rightarrow \infty} r^{n-2} u_{1}(r)=a \text { if } q>\frac{n}{n-2} ; \\
\lim _{r \rightarrow \infty} \frac{r^{n-2}}{\log r} u_{1}(r)=a \text { if } q=\frac{n}{n-2} ; \\
\lim _{r \rightarrow \infty} r^{q(n-2)-2} u_{1}(r)=a \text { if } q<\frac{n}{n-2} .
\end{gathered}
$$

In the latter case the constants $a$ and $b$ are related by

$$
b^{q}=a\left(q\left(n^{2}-4\right)-2 n-q^{2}(n-2)^{2}\right) .
$$

The extension to $q \geq \frac{n+2}{n-2}$ follows from interchanging the role of $u$ and $v$ and of $p$ and $q$.

In order to find the $\epsilon$-dependences of the terms in (3.12), (3.14) and (3.16), we need to consider three cases.

Case I. $\frac{n}{n-2}<q \leq \frac{n+2}{n-2}, \alpha \in\left[0, \frac{n-2}{2(n-1)}\right)$. As $\epsilon \rightarrow 0$ we have

$$
\begin{array}{r}
\left\|u_{\epsilon}\right\|_{1} \approx \epsilon^{\frac{n}{q+1}}=\epsilon^{\frac{n-2+2 \alpha}{2}}, \quad\left\|v_{\epsilon}\right\|_{1} \approx \epsilon^{\frac{n}{p+1}}=\epsilon^{\frac{n-2-2 \alpha}{2}} \\
\left\|u_{\epsilon}^{p}\right\|_{1} \approx \epsilon^{\frac{n}{p+1}}=\epsilon^{\frac{n-2-2 \alpha}{2}}, \quad\left\|v_{\epsilon}^{q}\right\|_{1} \approx \epsilon^{\frac{n}{q+1}}=\epsilon^{\frac{n-2+2 \alpha}{2}} \\
\left\|u_{\epsilon}\right\|_{L^{\infty}(\partial \Omega)} \approx \epsilon^{\frac{n}{q+1}}=\epsilon^{\frac{n-2+2 \alpha}{2}}, \quad\left\|v_{\epsilon}\right\|_{L^{\infty}(\partial \Omega)} \approx \epsilon^{\frac{n}{p+1}}=\epsilon^{\frac{n-2-2 \alpha}{2}},
\end{array}
$$


and

$$
\begin{aligned}
& n>4 \Rightarrow\left\|u_{\epsilon}\right\|_{2}^{2} \approx \epsilon^{n-\frac{2 n}{p+1}}=\epsilon^{2+2 \alpha}, \quad\left\|v_{\epsilon}\right\|_{2}^{2} \approx \epsilon^{n-\frac{2 n}{q+1}}=\epsilon^{2-2 \alpha}, \\
& n=4 \quad \Rightarrow \quad\left\|u_{\epsilon}\right\|_{2}^{2} \approx \epsilon^{2+2 \alpha}|\log \epsilon|, \quad\left\|v_{\epsilon}\right\|_{2}^{2} \approx \epsilon^{2-2 \alpha}|\log \epsilon|, \\
& n<4 \Rightarrow\left\|u_{\epsilon}\right\|_{2}^{2} \approx \epsilon^{2(n-2)-\frac{2 n}{p+1}}=\epsilon^{n-2+2 \alpha}, \quad\left\|v_{\epsilon}\right\|_{2}^{2} \approx \epsilon^{2(n-2)-\frac{2 n}{q+1}}=\epsilon^{n-2-2 \alpha}, \\
& n>6+2 \alpha \quad \Rightarrow \quad\left\|u_{\epsilon}\right\|_{\frac{p+1}{p}}^{2} \approx \epsilon^{4+4 \alpha}, \\
& n=6+2 \alpha \quad \Rightarrow \quad\left\|u_{\epsilon}\right\|_{\frac{p+1}{p}}^{2} \approx \epsilon^{4+4 \alpha}|\log \epsilon|^{\frac{2 p}{p+1}}=\epsilon^{2(n-4)}|\log \epsilon|^{2 \frac{n-2}{n}}, \\
& n<6+2 \alpha \quad \Rightarrow \quad\left\|u_{\epsilon}\right\|_{\frac{p+1}{p}}^{2} \approx \epsilon^{n-2+2 \alpha}, \\
& n>6-2 \alpha \Rightarrow\left\|v_{\epsilon}\right\|_{L}^{2} \frac{q+1}{q} \approx \epsilon^{4-4 \alpha}, \\
& n=6-2 \alpha \quad \Rightarrow \quad\left\|v_{\epsilon}\right\|_{L \frac{q+1}{q}}^{2} \approx \epsilon^{4-4 \alpha}|\log \epsilon|^{\frac{2 q}{q+1}}=\epsilon^{2(n-4)}|\log \epsilon|^{2 \frac{n-2}{n}}, \\
& n<6-2 \alpha \quad \Rightarrow \quad\left\|v_{\epsilon}\right\|_{L \frac{q+1}{q}}^{2} \approx \epsilon^{n-2-2 \alpha} .
\end{aligned}
$$

Here $f \approx g$ means that the quotient of $f$ and $g$ is bounded away from zero and infinity. All the asymptotic estimates above are consequences of the exact decay rates of $u_{1}$ and $v_{1}$ combined with the observation that for $\beta>0$ and $s \geq 1$ we have

$$
F(x) \approx|x|^{-\beta} \text { as }|x| \rightarrow \infty \Rightarrow \int_{\Omega} F\left(\frac{x}{\epsilon}\right)^{s} d x \approx\left\{\begin{array}{l}
\epsilon^{\beta s} \text { if } \beta s<n, \\
\epsilon^{n}|\log \epsilon| \text { if } \beta s=n, \\
\epsilon^{n} \text { if } \beta s>n .
\end{array}\right.
$$

We can now check for which values of $n, \alpha, \mu$ and $\lambda$ the maximum of $f^{*}\left(t w_{1}, s w_{2}\right)$ along (3.3) can be pushed below the critical Palais-Smale level by making $\epsilon$ small. When $\mu>0$ we use the second term on the right hand side of (3.14) to effect this. For the case $\mu=0$ we shall use the first term. First we assume that $n>4$ and $\mu>0$. We have

$$
-\frac{\lambda t^{2}}{2} \int_{\Omega} u_{\epsilon}^{2}-\frac{\mu s^{2}}{2} \int_{\Omega} v_{\epsilon}^{2} \approx-\lambda \epsilon^{2+2 \alpha}-\mu \epsilon^{2-2 \alpha} .
$$

The contribution of all the other terms in (3.12), (3.14) and (3.16) is seen from the asymptotics above to be at most the asymptotics of $\left\|v_{\epsilon}\right\|_{\frac{q+1}{q}}^{2}$. Thus, since $n>4$ and $\mu>0$, the second term in (4.2) dominates as $\epsilon \rightarrow 0$. If $\alpha>0, \mu=0$ and $\lambda>0$, the first term in (4.1) dominates (note there are fewer terms to consider) provided $2+2 \alpha<n-2$, i.e. $n>4+2 \alpha$.

For $n=4$ and $\alpha>0,(4.2)$ contains a logarithmic term. In this case we have to assume $\mu>0$. If $\alpha=0$ we need $\lambda+\mu>0$ (for all $n \geq 4$ ). Summarizing, the maximum along (3.3) may be pushed below the critical Palais-Smale level in each of the following cases:

$$
\alpha=0: \quad \lambda+\mu>0
$$




$$
0<\alpha<\frac{n-2}{2(n-1)}: \quad\left\{\begin{array}{l}
\mu>0, \\
n>4+2 \alpha, \mu=0, \lambda>0 .
\end{array}\right.
$$

Case II. $1<q<\frac{n}{n-2}, \alpha \in\left[\frac{n-2}{2(n-1)}, 1\right)$. We only have to consider the behaviour of terms with asymptotics differing from the asymptotics in Case I. These are given below:

$$
\begin{gathered}
\left\|u_{\epsilon}\right\|_{1} \approx\left\|v_{\epsilon}^{q}\right\|_{1} \approx\left\|u_{\epsilon}\right\|_{L^{\infty}(\partial \Omega)} \approx \epsilon^{\frac{q n}{p+1}}=\epsilon^{\frac{(n+2-2 \alpha)(n-2-2 \alpha)}{2(n-2+2 \alpha)}} ; \\
n<6 \alpha+2 \quad \Rightarrow \quad\left\|u_{\epsilon}\right\|_{2}^{2} \approx \epsilon^{\frac{2 q n}{p+1}}=\epsilon^{\frac{(n+2-2 \alpha)(n-2-2 \alpha)}{n-2+2 \alpha}} ; \\
n=6 \alpha+2 \quad \Rightarrow \quad\left\|u_{\epsilon}\right\|_{2}^{2} \approx \epsilon^{2+2 \alpha}|\log \epsilon| ; \\
n>6 \alpha+2 \quad \Rightarrow \quad\left\|u_{\epsilon}\right\|_{2}^{2} \approx \epsilon^{2+2 \alpha} ; \\
q(n-2)>\frac{n}{q+1} \quad \Rightarrow \quad\left\|u_{\epsilon}\right\|_{\frac{p+1}{p}}^{2} \approx \epsilon^{\frac{2 n p}{p+1}}=\epsilon^{n+2+2 \alpha} ; \\
q(n-2)=\frac{n}{q+1} \quad \Rightarrow \quad\left\|u_{\epsilon}\right\|_{\frac{p+1}{p}}^{2} \approx \epsilon^{n+2+2 \alpha}|\log \epsilon| ; \\
q(n-2)<\frac{n}{q+1} \Rightarrow\left\|u_{\epsilon}\right\|_{\frac{p+1}{p}}^{2} \approx \epsilon^{-\frac{2 n}{p+1}+2(q(n-2)-2)} \leq \epsilon^{q(n-2)-2}=\epsilon^{\frac{n(n-2-2 \alpha)}{n-2+2 \alpha}} .
\end{gathered}
$$

Proceeding as before, we use the term

$$
-\frac{\lambda t^{2}}{2} \int_{\Omega} u_{\epsilon}^{2}-\frac{\mu s^{2}}{2} \int_{\Omega} v_{\epsilon}^{2}
$$

to push the maximum along (3.3) down. The contributions of all the other terms in (3.12), (3.14) and (3.16) are seen from the above asymptotics to be at most the asymptotics of $\left\|v_{\epsilon}\right\|_{\frac{q+1}{q}}^{2}$. In particular, this term still dominates $\left\|u_{\epsilon}\right\|_{\frac{p+1}{p}}^{2}$.

Thus, for $\mu>0$ everything is the same as before, because the orders have not changed. However for $\mu=0$ we have to use $\left\|u_{\epsilon}\right\|_{2}^{2}$, which now has different asymptotics. Comparing with the remaining terms, we find that it dominates provided $q+1>p-1$. Summarizing, in terms of $n$ and $\alpha$, the maximum can be pushed below the critical Palais-Smale level in the following two cases:

$$
\left\{\begin{array}{l}
\mu>0 \\
n(n-4 \alpha-4)+4-4 \alpha^{2}>0, \mu=0, \lambda>0 .
\end{array}\right.
$$

The second condition on $n$ and $\alpha$ is certainly satisfied when $n \geq 4+4 \alpha$.

Case III. $q=\frac{n}{n-2}, \alpha=\frac{n-2}{2(n-1)}$. This case differs from the first because of the logarithmic term in the asymptotics for $u_{1}$. We now use

$F(x) \approx|x|^{-\beta} \log |x|$ as $|x| \rightarrow \infty \Rightarrow \int_{\Omega} F\left(\frac{x}{\epsilon}\right)^{s} d x \approx\left\{\begin{array}{l}\epsilon^{\beta s}|\log \epsilon|^{s} \quad \text { if } \beta s<n, \\ \epsilon^{n}|\log \epsilon|^{s+1} \quad \text { if } \beta s=n, \\ \epsilon^{n} \text { if } \beta s>n .\end{array}\right.$ 
Again we only give the behaviour of terms with asymptotics differing from the asymptotics in Case I:

$$
\begin{gathered}
\left\|u_{\epsilon}\right\|_{1} \approx\left\|v_{\epsilon}^{q}\right\|_{1} \approx\left\|u_{\epsilon}\right\|_{L^{\infty}(\partial \Omega)} \approx \epsilon^{\frac{n(n-2)}{2(n-1)}}|\log \epsilon| \\
n>4 \quad \Rightarrow \quad\left\|u_{\epsilon}\right\|_{L^{2}}^{2} \approx \epsilon^{\frac{3 n-4}{n-1}} ; \\
n=4 \quad \Rightarrow \quad\left\|u_{\epsilon}\right\|_{L^{2}}^{2} \approx \epsilon^{\frac{3 n-4}{n-1}}|\log \epsilon|^{3} \\
\left\|u_{\epsilon}\right\|_{\frac{p+1}{p}}^{2} \approx \epsilon^{2 \frac{3 n-4}{n-1}}
\end{gathered}
$$

For $\mu>0$ everything is as before. When $\mu=0$ and $\lambda>0$ we find that we need $n^{2}-6 n+6>0$.

End of the proof of Theorem 2. We define

$$
\Gamma=\left\{\gamma \in C\left([0,1] ; X_{\alpha}^{*}\right): \gamma(0)=(0,0), \gamma(1)=\left(t_{1} u_{\epsilon}^{p}, s_{1} v_{\epsilon}^{q}\right)\right\},
$$

with $t_{1}$ and $s_{1}$ satisfying (3.3). We choose $t_{1}$ and $s_{1}$ sufficiently large to ensure that $f^{*}(\gamma(1))<0$ for all sufficiently small $\epsilon>0$. In view of the analysis above we now know that for all sufficiently small $\epsilon>0$, we have

$$
0<c=\inf _{\gamma \in \Gamma} \max _{0 \leq t \leq 1} f^{*}\left(\gamma(t)<\frac{2}{n} K_{p, q}^{\frac{n}{2}} .\right.
$$

Thus the Palais-Smale condition holds (section 2) and $c$ is a critical value of $f^{*}$. As we have seen in section 1 , such a critical value is also a critical value of $f$. This yields the existence of a nontrivial solution.

Finally, in order to show that problem $\left(I_{p, q}\right)$ has a positive solution under the additional assumption $\lambda \mu<\lambda_{1}^{2}$, as in [AS] we just need to consider the modified Hamiltonian given by

$$
H_{\delta}(z)=\frac{\delta}{p+1}\left|u_{-}\right|^{p+1}+\frac{1}{p+1}\left|u_{+}\right|^{p+1}+\frac{\delta}{q+1}\left|v_{-}\right|^{q+1}+\frac{1}{q+1}\left|v_{+}\right|^{q+1} .
$$

For $\delta \in(0,1]$ one obtains exactly as before a nontrivial critical point $z_{\delta}$ corresponding to a positive critical value $c_{\delta}$ below the critical Palais-Smale level. This critical value is bounded away from zero and $\frac{2}{n} K_{p, q}^{\frac{n}{2}}$, uniformly in $\delta$. Therefore we may adjust the argument used in section 2 and extract a convergent sequence $z_{\delta_{n}}$. The limit is then a nontrivial solution of

$$
\begin{cases}-\Delta v=\lambda u+u_{+}^{p}, & \text { in } \Omega, \\ -\Delta u=\mu v+v_{+}^{q}, & \text { in } \Omega, \\ u=v=0, & \text { on } \partial \Omega,\end{cases}
$$

The regularity of weak solutions follows immediately from [HV1, section 5]. We emphasize that positivity of the solutions is not needed for the regularity results in [HV1], which are valid in the subcritical and critical case. By the maximum principle proved in [FM2], this solution is positive. This completes the proof of Theorem 2. 


\section{ACKNOWLEDGMENT}

Joost Hulshof and Enzo Mitidieri gratefully acknowledge the support from the EC through the project "Reaction Diffusion Equations", grant no. ERBCHRXCT930409, and MURST (60\%). The authors also acknowledge the hospitality of their institutions, which made this collaboration possible.

\section{REFERENCES}

[AR] A. Ambrosetti \& P.H. Rabinowitz, Dual variational methods in critical point theory and applications, J. Funct. Anal. 14, 349-381, 1973. MR 51:6412

[AS] A. Ambrosetti \& M. Struwe, A note on the problem $-\Delta u=\lambda u+u|u|^{2^{*}-1}$, Manuscripta Math. 54, 373-379, 1986. MR 87h:35076

$[\mathrm{BF}] \quad$ V. Benci \& D. Fortunato, The dual method in critical point theory - multiplicity results for strongly indefinite functionals, Ann. Mat. Pura Appl. (4) 134, 215-242, 1982. MR 84g:58025

[BR] V. Benci \& P.H. Rabinowitz, Critical point theorems for indefinite functionals, Invent. Math. 52, 241-273, 1979. MR 80i:58019

[BrL] H. Brezis \& E. Lieb, A relation between pointwise convergence of functions and convergence of functionals, Proc. Amer. Math. Soc. 88, 486-490, 1983. MR 84e:28003

[BrN] H. Brezis \& L. Nirenberg, Positive solutions of nonlinear elliptic equations involving critical Sobolev exponents, Comm. Pure Appl. Math. 36, 437-478, 1983. MR 84h:35059

[CE] F.H. Clarke \& I. Ekeland, Hamiltonian trajectories having prescribed minimal period, Comm. Pure Appl. Math. 33, 103-116, 1980. MR 81e:70017

[CFM1] Ph. Clément, D.G. de Figueiredo \& E. Mitidieri, Positive Solutions of Semilinear Elliptic Systems, Comm. Partial Diff. Equations, 17, 923-940, 1993. MR 93i:35054

[CFM2] Ph. Clément, D.G. de Figueiredo \& E. Mitidieri, A priori estimates for positive solutions of semilinear elliptic systems via Hardy-Sobolev inequalities, Nonlinear Partial Differential Equations (Fès, 1994), Pitman Res. Notes in Math. Ser., vol. 343, Longman Sci. Tech., Harlow, 1996, pp. 73-91. CMP 97:03

[CV1] Ph. Clément \& R.C.A.M. vanderVorst, Interpolation spaces for $\partial_{T}$-systems and applications to critical point theory, Panamer. Math. J. 4, 1-45, 1994. MR 96a:58075

[CV2] Ph. Clément \& R.C.A.M.vanderVorst, On a semilinear elliptic system, Diff. \& Int. Eq. 8, 1317-1329, 1995. MR 96e:35043

[FF] P. Felmer \& D.G. de Figueiredo, On superquadratic elliptic systems, Trans. Amer. Math. Soc. 343, 99-116, 1994. MR 94g:35072

[FM1] D.G. de Figueiredo \& E. Mitidieri, A maximum principle for an elliptic system and applications to semilinear problems, SIAM J. Math. Anal 17, 836-849, 1986. MR 87h:35111

[FM2] D.G. de Figueiredo \& E. Mitidieri, Maximum principles for cooperative systems, C.R. Acad. Sci. Paris Sér. I Math. 310, 49-52, 1990. MR 91e:35040

[Ho] H. Hofer, On strongly indefinite functionals with applications, Trans. Amer. Math. Soc., 275, 1, 185-214, 1983. MR 84c:58015

[HV1] J. Hulshof \& R.C.A.M. vanderVorst, Differential systems with strongly indefinite variational structure, J. Funct. Anal. 114(1), 32-58, 1993. MR 94g:35073

[HV2] J. Hulshof \& R.C.A.M. vanderVorst, Asymptotic behaviour of ground states, Proc. Amer. Math. Soc. 124 (1996), 2423-2431. MR 96m:35082

[L1] P.L. Lions, The concentration-compactness principle in the calculus of variations. The Limit case I, Rev. Mat. Iberoamericana 1 (1), 145-201, 1985. MR 87c:49007

[L2] P.L. Lions, The concentration compactness principle in the calculus of variations. The Limit case II, Rev. Mat. Iberoamericana 1 (2), 45-121. MR 87j:49012

[L3] P. L. Lions, On the existence of positive solutions of semilinear elliptic equations, SIAM Review 24, 441-467, 1982. MR 84a:35093

[MM] G. Mancini \& E. Mitidieri, Positive solutions of some coercive-anticoercive elliptic systems, Ann. Fac. Sci. Toulouse VIII (3), 257-292, 1986. MR 89h:35023

[M] E. Mitidieri, A Rellich type identity and applications, Comm. Partial Diff. Equations 18 (1 \& 2), 125-151, 1993. MR 94c:26016

[P] S.I. Pohožaev, On the eigenfunctions of quasi-linear elliptic problems, Math. USSR Sb. 11, 171-188, 1970. MR 42:8081 
[PS] P. Pucci \& J. Serrin, A general variational identity, Indiana Univ. Math. J. 35, 681-703, 1986. MR 88b:35072

[PV] L.A. Peletier \& R. C. A. M. vanderVorst, Existence and non-existence of positive solutions of nonlinear elliptic systems and the biharmonic equation, Differential \& Integral Equations, 54, 747-767, 1991. MR 93c:35039

[V1] R.C.A.M. vanderVorst, Variational identities and applications to differential systems, Arch. Rat. Mech. Anal. 116, 375-398, 1991. MR 93d:35043

[V2] R.C.A.M. vanderVorst, Fourth order elliptic equations with critical growth, C.R. Acad. Sci. Paris Sér. I Math. 320, 295-299, 1995. MR 96b:35076

Mathematical Institute, Leiden University, P.O. Box 9512, 2300 RA Leiden, The NETHERLANDS

E-mail address: hulshof@wi.leidenuniv.nl

URL: http://www.wi.leidenuni.nl/home/hulshof/

Dipartimento di Scienze Matematiche, Università degli Studi di Trieste, Piazzale Europa 1, 34100 Trieste, Italy

Center for Dynamical Systems and Nonlinear Studies, Georgia Institute of TechNology, Atlanta, Georgia 30332-0190

E-mail address: rvander@math.gatech.edu

$U R L:$ http://www.math.gatech.edu/rvander/ 